\title{
SYNERGISTIC GROWTH EFFECT AMONG BACTERIA RECOVERED FROM ROOT CANAL INFECTIONS
}

\author{
Gil Moreira Júnior' ${ }^{1}$, Antônio Paulino Ribeiro Sobrinho ${ }^{2 *}$, Bernardo Henrique Silva Bambirra ${ }^{2}$, Felipe Henrique Silva \\ Bambirra $^{3}$, Maria Auxiliadora Roque Carvalho ${ }^{4}$, Luiz Macedo Farias ${ }^{6}$, Jacques Robert Nicoli ${ }^{4}$, Elizabeth Spangler \\ Moreira $^{4}$
}

${ }^{1}$ Faculdade de Odontologia, Universidade de Itaúna, Itaúna, MG, Brasil; ${ }^{2}$ Faculdade de Odontologia, Universidade Federal de Minas Gerais, Belo Horizonte, MG, Brasil; ${ }^{3}$ Faculdade de Terapia Física, Universidade Federal de Minas Gerais, Belo Horizonte, MG, Brasil; ${ }^{4}$ Departamento de Microbiologia, Instituto de Ciências Biológicas, Universidade Federal de Minas Gerais, Belo Horizonte, MG, Brasil.

Submitted: February 26, 2010; Approved: March 14, 2011.

\begin{abstract}
The objective of this study was to determine the ecological relationships between bacterial species that colonize infected root canals. Root canal bacteria recovered from one patient with pulp canal necrosis were evaluated in vitro for synergistic and antagonistic activities determined by mono and co-culture growth kinetics and the production of bacteriocin-like substances using the double layer diffusion method.

Peptostreptococcus prevotii triggered a significant increase of Fusobacterium nucleatum growth, while the former bacteria did not affect the growth of $P$. prevotii. The bacterial species did not produce antagonism activity against itself or against any of the other two species. Despite many studies have demonstrated the capability of root canal microorganisms to produce antagonistic substances, these in vitro experimental tests show the synergistic effect of $P$. prevotii on the growth of $F$. nucleatum.
\end{abstract}

Keywords: Root canal, infection, bacterial relationships.

\section{INTRODUCTION}

Microorganisms that inhabit the oral cavity may infect necrotic root canal systems (RCS) and induce inflammatory processes in adjacent periapical tissues. The microorganisms present in these infections belong to a limited group when compared to the potential candidates present in the oral cavity $(18,19)$, suggesting that some species are more adapted than others to colonize these sites $(29,30)$. There is a predominance of anaerobic species in these infections, and the majority of them can also be recovered from the periodontal pockets (36).

Significant differences have been observed in the composition of pathogenic microbiota present in symptomatic and asymptomatic cases, suggesting that the structure of the bacterial community might determine clinical symptomatology $(5,25,26,35)$. Other researches did not find differences in the composition of the microbiota when comparing clinical symptomatology $(2,15)$.

\footnotetext{
*Corresponding Author. Mailing address: Departamento de Odontologia Restauradora, Faculdade de Odontologia, Universidade Federal de Minas Gerais, Belo Horizonte, MG, Brazil, Av. Antonio Carlos 6627, CEP 31270-901, Belo Horizonte, MG, Brazil.; Tel. 55-31-3409.2470.; E-mail: sobrinho.bhz@ terra.com.br
} 
To date, although many studies have characterized the diversity of this microbiota, few of them have been focused on ecological relationships. Factors such as the oxygen balance, temperature, availability of nutrients, and bacterial interactions appear to influence the dynamic growth and colonization of the infected RCS $(5,6,28,29,30)$.

Microbial interactions, which can be cooperative or interfering, frequently defined as synergism or antagonism relationships, have long been recognized as important determinants of ecological niches. Interspecies cooperation occurs by hydrolysis of complex carbohydrates and sequential fermentation of the resulting sugars, as well as by the exchange of growth factors. Common interference mechanisms include the competition for nutrients and adhesion sites, as well as the production of toxic metabolites and specific antimicrobial compounds, such as bacteriocins. Bacteriocins are bactericide proteins once thought to be effective only among closely related strains, but broader activity and selective action against distant relatives also occurs (33). Additionally, they may influence the organization of the biofilm that forms inside the infected RCS by inhibiting the growth of some species that compete for the same ecological niche $(16,27)$. Bacteriocin produced by strains of Lactococcus lactis has been used as a food preservative and evaluated as an antimicrobial agent to control dental plaque (13) as well as an endodontic antimicrobial solution (34). Although still relatively few characterized, it is known that bacteriocins are produced by oral microorganisms.

As the previous literature has rarely assessed microbial interactions in RCS infections, the present study attempted to determine possible ecological factors involved in microbial root canal colonization. We assayed synergistic and antagonistic relationships among strains recovered from one patient with pulpal necrosis (15) as well as their capability to produce bacteriocin-like substances.

\section{MATERIALS AND METHODS}

\section{Bacterial strains}

The microorganisms used in this investigation were recovered from one patient with necrotic pulp infection treated at the Endodontic Clinic of the Dental School at the Universidade Federal de Minas Gerais (15). The following microbial species were selected: Fusobacterium nucleatum, Lactobacillus paracasei and Peptostreptococcus prevotii.

\section{"In vitro" assay for production of antagonistic substances}

The selected species L. paracasei, $F$. nucleatum and $P$. prevotii were tested for its inhibition activity against itself as well as against the other species (Clostridium butyricum, Prevotella intermedia, Streptococcus anginosus, and Gemella morbillorum) also recovered from human necrotic pulp infection (15), using the double layer method (31). The $F$. nucleatum and $P$. prevotii strains were grown in BHI-S (Difco) and L. paracasei in de Man, Rogosa and Sharp broth (MRS, Difco), and incubated at $37^{\circ} \mathrm{C}$, for 24 hours (L. paracasei) and 48 hours ( $P$. prevotti and F. nucleatum) in an anaerobic chamber (Forma Scientific Company, Marietta, $\mathrm{OH}$, USA), containing an atmosphere of N2 85\%, H2 10\% and $\mathrm{CO} 25 \%$. Then, spots of $5 \mu \mathrm{l}$ of the cultures were made onto BHI$\mathrm{S}$ agar. The plates were incubated for periods of 24 hours and 48 hours, respectively, under the same anaerobic conditions. After incubation the plates were removed from the anaerobic chamber, exposed to chloroform vapor for 30 minutes and left open inside a laminar flow chamber for an equal period of time to allow evaporation of residual Chloroform. Plates were covered with 3.5 $\mathrm{ml}$ of soft agar $(0.75 \%$ BHI-S or $0.75 \%$ MRS according to indicator species), inoculated with approximately $10^{7}$ colony forming units (CFU) of the indicator bacteria and incubated under the specific cultivation conditions for each one. The presence of an inhibition zone around the spot indicated the production of antagonist substances. Diameter of the inhibition halo was measured using a digital pachymeter (Mitutoyo Sul América Ltda, Suzano, SP, Brazil). Experiments were done in duplicate.

\section{Growth Curve Assay for the determination of bacterial synergistic relationship.}

To ascertain in vitro synergism or antagonistic relationships between the microorganisms, individual and associated growth curves were determined for L. paracasei, $F$. nucleatum and $P$. prevotii. Associated growth curves were 
established analyzing the three isolates in pairs. For inoculum standardization, individual growth curve of each microorganism were previously determined by measuring absorbance at different times of incubation using a spectrophotometer (Spectrum series SP-2100, Hangzhou, China) at $600 \mathrm{~nm}$ and by evaluating the number of Colony Forming Unit (CFU) per $\mathrm{ml}$, to find the time range for logarithmic phase of each microorganism. Individual and cocultures were then carried out using as inocula similar populations in their log phase as previously determined, to evaluate neutral, synergistic or antagonistic relationships, comparing the growth curve profile of each bacterium in pure culture with that obtained when co-cultured. Pure cultures were plated onto brain heart infusion (Difco, Sparks, MD, USA) agar supplemented with hemin and menadione (S-BHA), without antibiotics (control experiments). Organisms were cocultured in supplemented BHI (S-BHI) and subsequently evaluated in respect to growth stimuli by plating to obtain bacterial counts onto S-BHI agar (S-BHA) containing an antibiotic selected to inhibit the growth of only one of the two microorganisms. For L. paracasei-P. prevotii co-culture, metronidazol (Sigma, St. Louis, LO, USA) was added to the SBHA at a concentration of $1 \mu \mathrm{g} / \mathrm{ml}$ for selectively count of $L$. paracasei. For L. paracasei-F. nucleatum co-culture, amoxicillin trihydrate (Glaxo SmithKline, Rio de Janeiro, Brazil) and potassium clavulanate (Glaxo SmithKline) at 0.06 $\mu \mathrm{g} / \mathrm{ml}$ were supplemented to BHI-S agar for L. paracasei count. For $P$. prevotii- $F$. nucleatum co-culture, erythromycin (Sigma) and chloramphenicol (Inlab, São Paulo, SP, Brazil) were added to the plate at $0.06 \mu \mathrm{g} / \mathrm{ml}$ to inhibit $F$. nucleatum and $P$. prevotii growth, respectively. Cultures were incubated in an anaerobic chamber containing an atmosphere of $\mathrm{N}_{2}, 10 \%$ $\mathrm{H}_{2}$ and $5 \% \mathrm{CO}_{2}$ (Forma Scientific Inc., Marietta, OH, USA) at $37^{\circ} \mathrm{C}$. At different periods of time culture samples (pure and associated) were removed and submitted to successive decimal dilutions. Cell growth was determined by counting the number of CFU/ml until 72 hours of culture.

\section{Statistical analysis}

Data were subjected to the normalization test (ShapiroWilk) and, subsequently, they were analyzed using an unpaired (Student's $t$ test) test $(\mathrm{p}<0.05)$.

\section{RESULTS}

\section{Antagonistic assay}

The selected bacterial species $F$. nucleatum and $P$. prevotii did not produce antagonism activity against itself or against any of the bacteria tested. L. paracasei inhibited the growth of S.anginosus and G. morbillorum (Table 1).

Table 1. Cross-test for presence (+) of bacteriocin-like activities between the selected bacteria.

\begin{tabular}{l|l|l|l|l|l|l|l}
\hline Producing Strain & \multicolumn{7}{c}{ Target Strain } \\
& A & B & C & D $^{*}$ & $\mathbf{E}^{*}$ & F $^{*}$ & G $^{*}$ \\
\hline P. prevotii (A) & - & - & - & - & - & - & - \\
\hline F. nucleatum (B) & - & - & - & - & - & - & - \\
\hline L. paracasei (C) & - & - & - & - & - & + & + \\
\hline${ }^{*}$ D- C. butyricum, E - P. intermedia, F - S. anginosus, G - G. morbillorum
\end{tabular}

+ Presence of antagonism; - absence of antagonism

\section{Growth curves assay}

In pure culture $F$. nucleatum showed a log phase occurring in the period of 12-48 hours of incubation and reaching a final cell density of $10^{7} \mathrm{CFU} / \mathrm{ml}$ (Figure 1A). For P. prevotii a maximal cell density of about $10^{8} \mathrm{CFU} / \mathrm{ml}$ was observed after 36 hours of incubation (Figure 1B). A short log phase (between 0 to 12 hours of incubation) was detected for $L$. paracasei, as can be seen in Figure 2.

The presence of $P$. prevotii triggered a significant increase in the growth of $F$. nucleatum after 60 hours of incubation (Fig. $1 \mathrm{~A}, \mathrm{p}<0.05)$. Therefore, co-culture did not affect the growth of $P$. prevotii, as demonstrated in Fig 1B ( $>>0.05)$.

Regarding the co-culture of L. paracasei and F. nucleatum (Fig. 2A), no statistically significant difference for growth of $L$. paracasei $(\mathrm{p}>0.05)$ was observed when compared to its pure 
culture. The influence of $L$. paracasei on the growth of $F$. nucleatum was not evaluated in this study because no antibiotic concentration could be found that inhibited L. paracasei without influencing $F$. nucleatum.

Co-culture of $L$. paracasei and $P$. prevotii also did not show significant influence for L. paracasasei growth $(\mathrm{p}<0.05)$ when compared to the pure culture (Fig. 2B), despite some statistically difference found in the graphic. Due to the inability to find a suitable antibiotic concentration, the effect of $P$. prevotii on L. paracasei was not assessed.

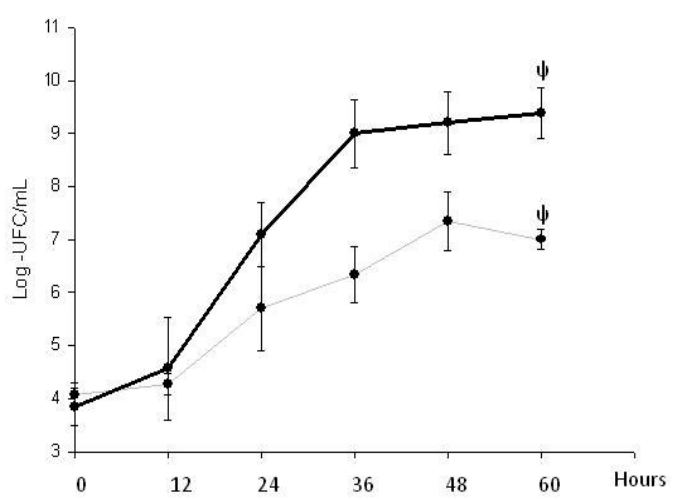

- GC - F nucleatum- pure culture

$\rightarrow$ GC - F. nucleatum - associated to P. prevotii

\section{A}

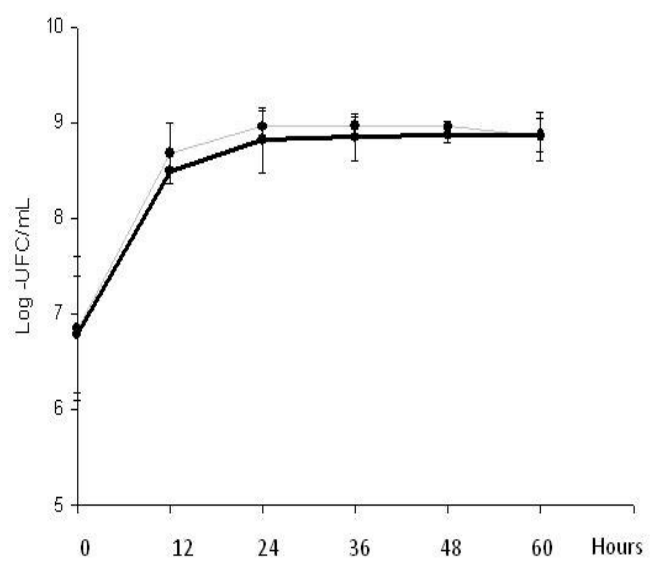

- GC-L paracase- pure culture

- GC- L paracasei- associated to F. nucleatum

\section{A}

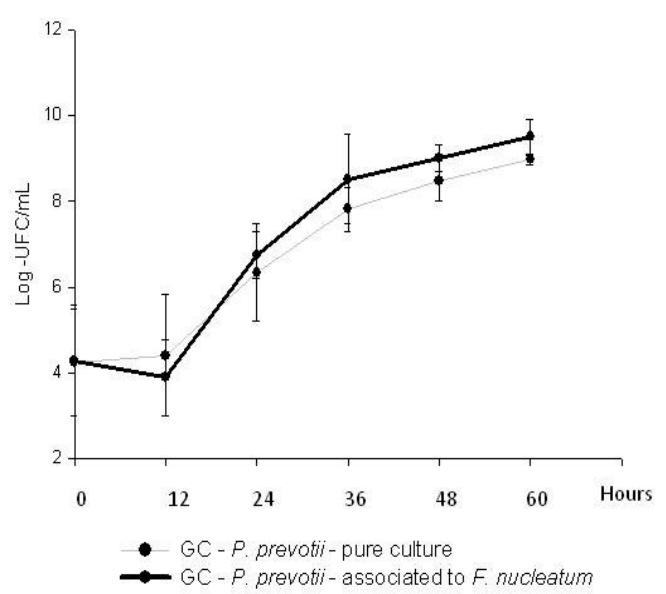

B

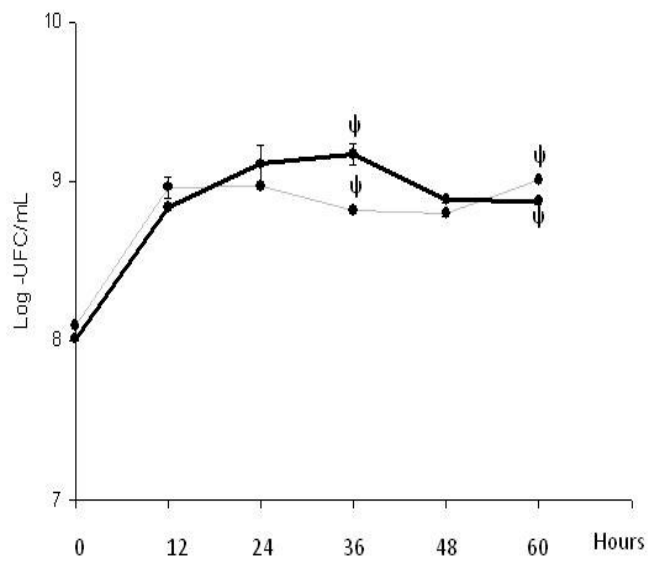

- GC - L paracasei-pure culture

* GC-L paracasei-associated to P. prevotii

Figure 1. (A) Growth curve for coculture between $F$. nucleatum and P. prevotii; (B) Growth curves for co-culture between $P$. prevotii and F. nucleatum. Comparisons between mono- and co-cultures were carried out as described in the Materials and Methods. Bars represent the means of three experiments, performed in duplicate, whereas the lines represent the standard errors of the mean. $\Psi$ : statistical difference (ttest) between mono and co-culture $(\mathrm{p}<0.05)$.

Figure 2. (A) Growth curve for coculture between $L$. paracase $i$ and $F$. nucleatum; (B) Growth curve for co-culture between $L$. paracasei and $P$. prevotii. Comparisons between mono- and co-cultures were carried out as described in the Materials and Methods. Bars represent the means of three experiments, performed in duplicate, whereas the lines represent the standard errors of the mean. $\Psi$ : statistical difference (ttest) between mono and co-culture $(\mathrm{p}<0.05)$. 


\section{DISCUSSION}

Microbial consortia play an important role in the etiology and pathogenesis of oral diseases, determining clinical parameters of inflammation and periodontal destruction (27, 28). Ecological factors such as synergistic and antagonistic interactions, co-aggregation, and availability of nutrients influence the composition of a given microbiota (10).

In root canal infections, specific bacterial species and combinations of species determine the composition of the microbial ecology, providing indispensable growth factors and physico-chemical changes in the environment $(28,29)$. Similarly, the evolution of clinical signs and symptoms as well as the destruction of apical bone can be influenced by relationships between bacterial species $(9,16,18)$. The present study assessed synergism and antagonism among microorganisms recovered from human root canal infections (15) as well as their capability to produce bacteriocin-like substances. Control reference strains (ATCC) were not assayed in this study, since recent clinical isolates apparently express virulence factors different from those observed in culture collection strains (1). Additionally, to evaluate synergistic effects the growth stimuli is better furnished by strains isolated from the same infected site.

Microbial relationships were analyzed through the comparison of growth curves of microorganisms in mono or co-cultures. Previous investigators have found that specific bacterial combinations play a role in the evolution of infectious processes, particularly in pulp and periapical diseases $(12,32)$. The presence of organisms such as Peptostreptococcus, Eubacterium, Fusobacterium, and some black pigmented Gram negative strict anaerobic rods have been associated with symptomatic root canal infections (4, 7, 20). Conversely, facultative anaerobes such as Streptococcus and enteric bacteria have been linked to asymptomatic infections $(4,12)$.

The results presented here showed that the growth of $P$. prevotii (Figure 1B) and L. paracasei (Figure 2A and 2B) were not altered by co-culture with $F$. nucleatum or $P$. prevotti, respectively. On the other hand, the synergistic effect of $P$. prevotii on the growth of $F$. nucleatum was demonstrated by the significant growth increase of $F$. nucleatum $(\mathrm{p}<0.05)$, (Figure 1A) when co-cultured. Although many studies have demonstrated the capability of root canal microorganisms to produce antagonistic substances $(4,8,22)$, this is the first study to show synergistic effect between Fusobacterium and Peptostreptococcus.

Several studies have been assayed bacterial antagonistic activity in root canal microbiota $(3,21)$. According to Klaenhammer (14), lactic acid bacteria produce antibacterial substances that vary in their spectrum of activity, mode of action, molecular mass, genetic determinants, and biochemical characteristics. Their actions can be auto - or heteroantagonistic and affect a wide variety of targets including Gram-negative bacteria (17). In this study, L. paracasei presented antagonistic activity against $S$. anginosus and $G$. morbillorum.

Ribeiro-Sobrinho et al. (22) reported strong autoantagonistic activity of $F$. nucleatum and against $G$. morbillorum. In the present study, F. nucleatum presented neither auto- nor hetero-antagonism toward tested bacteria. Oliveira et al. (16) also did not detect antagonistic activity of $F$. nucleatum toward $P$. intermedia, $P$. nigrescens or $P$. gingivalis. In a previous study, $F$. nucleatum recovered from the same infection (15) was shown to survive and translocate to the sub-mandibular lymph node of germ-free mice (22), to modulate the periapical response through the expression of a particular profile of cytokines in murine root canal infections (23), and to induce apoptosis in murine lymph node cells (24).

Most previous studies examining the ecological complexity of root canal infections have shown antagonistic bacterial activity in these sites $(2,3,16,21)$. The present study shows that synergism could also occur. However, these potential mechanisms described here and in all literature have been demonstrated by in vitro assays and such result can not be always extrapolated to in vivo environment. Further comprehensive microbiological studies of multiple samples and 
under in vivo conditions are necessary to better understand the ecological determinants that affect these infections, thus culminating in the development of new prevention and treatment procedures.

\section{ACKNOWLEDGMENTS}

The authors are grateful to the post-graduate program in Microbiology and to the Laboratory of Microbial Ecology and Oral Microbiology, UFMG for their support. The study was also supported by grants from Conselho Nacional de Pesquisa $(\mathrm{CNPq})$ and Fundação de Amparo à Pesquisa do Estado de Minas Gerais (FAPEMIG).

\section{REFERENCES}

1. Baumgartner, J.C.; Falker Jr., W.A.; Beckerman, T. (1992), Experimentally induced infection by oral anaerobic microorganisms in a mouse model. Oral Microbiol. Immunol. 7, 253 - 256.

2. Brito, L.C.N.; Teles, F.R.; Teles, R.P.; França, E.C.; Ribeiro Sobrinho, A.P.; Haffajee , A.D.; Socransky, S.S. (2007). Use of Multiple Displacement Amplification and Checkerboard DNA-DNA Hybridization to Examine the Microbiota of Endodontic Infections. $J$. Clin. Microbiol. 45 (9), 3039 - 3049.

3. Caires, N.C.M.; Matos, A.M.; Farias, L.M.R.; Carvalho, M.A.R.; Drummond, R.M.N.; Nicoli, J.R.; Ribeiro Sobrinho, A.P. (2007). Partial characterization of antagonistic substance produced by Clostridium butirycum strain. Braz. J. Microbiol. 38, 265 - 269

4. Chavez de Paz, L.C. (2002). Fusobacterium nucleatum in endodontic flare-ups. Oral Surg. Oral Med. Oral Pathol. 93, 179 - 183.

5. Chu Frederick, C.S.; Tsang, C.S.P.; Chow Tak, W.; Samaranayke, L.P. (2005). Identification of cultivable microrganisms from primary endodontic infections with exposed and unexposed pulp space. J. Endod. 31 (6), 424 - 429.

6. De Sanctis, M.; Vignoletti, G.; Chieff, S. (1991). Relazioni endoparodontali-aspetti microbiologici e clinici. Dent. Cadmos. 59, 36 - 42.

7. Gomes, B.P.F.A.; Drucker, D.B.; Lilley, J.D. (1994). Positive and negative associations between bacteria species in dental root canals. Microbios. 80 (325), $231-243$

8. Gomes, B.P.F.A.; Lilley, J.D.; Drucker, D.B. (1996). Association of endodontic symptoms and signs with particular combinations of specific bacteria. Int. Endod. J. 29, 69 - 75 .

9. Gomes, B.P.F.A.; Jacinto, R.C.; Pinheiro, E.T.; Sousa, E.L.R.; Zaia,
A.A.; Ferraz, C.C.R.; Souza Filho, F.J. (2005). Porphyromonas gingivalis, Porphyromonas endodontalis, Prevotella intermedia and Prevotella Nigrescens in endodontic lesions detected by culture and PCR. Oral Microbiol. Immunol. 20, 211 - 215.

10. Haffajje, A.D.; Socranscky, S.S. (2005). Microbiology of periodontal disease: introduction. Periodontol. 200038 (12), 9 - 12.

11. Jacinto, R.C.; Gomes, B.P.F.A.; Shah Haroun, N.; Ferraz, C.C.; Zaia, A,A.; Souza Filho, F.J. (2005). Quantification of endotoxins in necrotic root canals from symptomatic and asymptomatic teeth. J. Med. Microbiol. 54, 777 - 783.

12. Jacinto, R.C.; Gomes, B.P.F.A.; Ferraz, C.C.R.; Zaia, A.A.; Souza Filho, F.J. (2003). Microbiological analysis of infected root canals from symptomatic and asymptomatic teeth with periapical periodontitis and the antimicrobial susceptibility of some isolated anaerobic bacteria. Oral Microbiol. Immunol. 18, 285 - 292.

13. Jonhson, I.H.; Hayday, H.; Coleman, G. (1978). The effects of nisin on the microbial flora of dental plaque of monkeys (Macaca fascicularis). $J$. Appl. Bacteriol. 45 (1), 99 - 109.

14. Klaenhammer, T.R. (1988). Bacteriocins of latic acid bacteria. Biochimie $70,337-49$.

15. Lana, M.A.; Ribeiro Sobrinho, A.P.; Stehling, R.; Garcia, G.D.; Silva, B.K.C.; Hamdan, J.S.; Nicoli, J.R.; Carvalho, M.A.R.; de Farias, L.M. (2001). Microorganisms isolated from root canal presenting necrotic pulp and their drugs susceptibility in vitro. Oral Microbiol. Immunol. 16 (2), $100-105$.

16. Oliveira, A.A.P.; Farias, L.M.; Nicoli, J.R.; Costa, E.; Carvalho, M.A.R. (1998). Bacteriocion production by Fusobacterium isolates recovered from the oral cavity of human subjects with and without periodontal disease and marmosets. Res. Microbiol. 149, 585 - 594.

17. Oliveira, R.B. P.; Oliveira, A.L.; Glória, M.B.A. (2008). Screening of lactic acid bacteria from vaccum packaged beef for antimicrobial activity. Braz. J. Microbiol. 39, 368 - 374.

18. Paster, B.J.; Boches, S.K.; Galvin, J.L.; Ericson, R.E.; Lau, C.N.; Levanos, V.A.; Sahasrabudhe, A.; Dewhirst, F.E. (2001). Bacterial diversity in human subgingival plaque. Journal of Bacteriology 183, $3770-3783$.

19. Perea, J.E. (2004). La flora de la boca en la era de la biología molecular. Med. Oral Patol. Oral Cir. Bucal 9, 1 - 10.

20. Pinheiro, E.T.; Gomes, B.P.F.A.; Ferraz, C.C.R.; Sousa, E.L.R; Teixeira, F.R.; Souza Filho, F.J. (2003). Microorganisms from canals of root-filled teeth with periapical lesions. Int. Endod. J. 36, 1- 11.

21. Peters, L.B.; Wesselink, P.R.; Van Winkelhoff, A.J. (2002). Combinations of bacterial species in endodontic infections. Int. Endod. J. 35, $698-702$.

22. Ribeiro Sobrinho, A.P.; Almeida, L.M.; Farias, L.M.; Carvalho, M.A.R.; Nicoli, J.R.; Uzeda, M.; Vieira, L.Q. (2001). Implantation of bacteria from human pulpal necrosis and translocation from root canals in 
gnotobiotic mice. J. Endod. 27, 605 - 609.

23. Ribeiro Sobrinho, A.P.; Maltos, S.M.M.; Farias, L.M.; Carvalho M.A.R.; Nicoli, J.R.; Uzeda, M.; Vieira, L.Q. (2002). Cytokine production in response to endodontic infection in germ free animals. Oral microbial. Immunol. 17, 334 - 353.

24. Ribeiro Sobrinho, A.P.; Rabelo, F.L.A.; Farias; L.M.; Figueiredo, C.B.O.; Leite, J.I.A.; Nicoli, J.R.; Uzeda, M.; Vieira, L.Q. (2005). Bacteria recovered from dental pulp induce apoptosis of lymph node cells. J. Med. Microbial. 54, 413 - 416.

25. Sakamoto, M.; Rôças, I.N.; Siqueira Jr, J.F.; Benno, Y. (2006). Molecular analysis of bacteria in asymptomatic and symptomptomatic endodontic infections. Oral Microbiol. Immunol. 21, 112 - 122.

26. Siqueira Jr, J.F.; Rôças, I.N.; Rosado, A.S. (2004) Investigation of bacterial communities associated with asymptomatic and symptomatic endodontic infections by denaturing gradient gel electrophoresis fingerprinting approach. Oral Microbiol. Immunol. 19, 363 - 370.

27. Socransky, S.S.; Haffajje, A.D.; Cugini, M.A.; Smith ,C.; Kent Jr, R.L. (1998). Microbial complexes in subgengival plaque. J. Clin. Periodontol. $25,134-44$.

28. Socransky, S.S; Haffajee, A.D. (2005). Periodontal microbial ecology. Peridontol. 2000. 38, 135 - 187.
29. Sundquivst, G. (1994). Taxonomy, ecology, and pathogenicity of the root canal flora. Oral Surg. Oral Med. Oral Pathol. 78, 522 - 530.

30. Sundqvist, G. (1992). Association between microbial species in dental root canal infections. Oral Microbiol. and Immunol. 7, 257 - 262.

31. Taag, J.; Dagani, A.S.; Wannamaker, L.M. (1976). Bacteriocins of Gram positive bacteria. Bacteriol. Rev. 40, 722 - 756.

32. Tani-Ishii, N.; Wang, C.Y. Tanner, A.; Stashenko, P. (1994). Changes in root canal microbiota during the development of rat periapical lesions. Oral Microbiol. Immunol. 9, 129 - 135.

33. Todorov, S.D. (2009). Bacteriocins from Lactobacillus plantarum production, genetic organization and mode of action. Braz. J. Microbiol. $40,209-221$.

34. Turner, S.R.; Love, R.M.; Lyons, K.M. (2004). An in vitro investigation of the antibacterial effect of nisin in root canals and canal wall radicular dentine. Int. Endod. J. 37 (10), 664 - 671.

35. Yoshida, M.; Fukushima, H.; Yamamoto, K.; Ogawa, K. T.; Sagawa, H. (1987). Correlation between clinical symptom and microorganisms isolated from root canals of teeth with periapical pathosis. J. Endod. 13, $24-28$.

36. Zehnder, M.; Gold, S.I.; Hasselgren, G. (2002). Pathologic interactions in pulpal and periodontal tissues. J. Clin. Periodontol. 29, $663-671$. 